\title{
Atividade antifúngica do óleo essencial de Cymbopogon winterianus contra Candida não-albicans de importância clínica no atendimento pediátrico
}

\author{
Antifungal activity of the essential oil of Cymbopogon winterianus against Candida non-albicans of clinical \\ importance in pediatric care
}

Actividad antifúngica del aceite esencial de Cymbopogon winterianus contra Candida no albicans de importancia clínica en atendimiento de pediatria

Ana Luísa de Araújo LIMA ${ }^{1}$

Abrahão Alves de OLIVEIRA FILHO²

Ana Luíza Alves de Lima PÉREZ ${ }^{3}$

Janiere Pereira de SOUSA ${ }^{\mathbf{1}}$

Lilian Sousa PINHEIRO ${ }^{1}$

Hermes DINIZ NETO ${ }^{1}$

José Pinto de SIQUEIRA JÚNIOR ${ }^{1}$

Edeltrudes de Oliveira LIMA ${ }^{1}$

\begin{abstract}
${ }^{I}$ Departamento de Ciências Farmacêuticas, Centro de Ciências da Saúde, Universidade Federal da Paraíba (UFPB), 58059-900, João Pessoa - PB, Brasil ${ }^{2}$ Unidade Acadêmica de Ciências Biológicas, Universidade Federal de Campina Grande (UFCG) 58700-970 Patos-PB, Brasil

${ }^{3}$ Programa de Pós-graduação em Odontologia, Centro de Ciências da Saúde, Universidade Federal da Paraíba (UFPB), 58059-900, João Pessoa - PB, Brasil
\end{abstract}

\section{Resumo}

Introdução: Devido às altas taxas de mortalidade, a candidemia têm se tornado um grave problema de saúde na realidade pediátrica, sobretudo quando se considera o aumento de infecções e a letalidade dos casos em grupos de risco como as crianças hospitalizadas e com sistema imunológico deficiente. Ultimamente têm-se percebido um aumento de infecções fúngicas provocadas por espécies não-albicans, trazendo uma nova realidade para o combate destas infecções, especialmente por envolver espécies resistentes à terapia convencional. Já foi demonstrado que o óleo essencial de Cymbopogon winterianus possui uma vasta gama de propriedades farmacológicas, incluindo atividade antifúngica. Objetivo: Este trabalho buscou avaliar a atividade antifúngica do óleo essencial de Cymbopogon winterianus Jowitt ex Bor (Poaceae) contra isolados de Candida não-albicans de importância clínica pediátrica. Material e Método: A concentração inibitória mínima (CIM) e a concentração fungicida mínima (CFM) foram determinadas pelas técnicas de microdiluição em caldo. Resultados: Tanto a CIM 50 quanto a $\mathrm{CFM}_{50}$ do óleo essencial de $C$. winterianus para os isolados testados foi de $128 \mu \mathrm{g} / \mathrm{mL}$.Conclusão: Este produto natural apresentou potencial antifúngico in vitro contra cepas de Candida não-albicans clinicamente relevante para a pediatria médica. Contudo, mais estudos são necessários para elucidar seu mecanismo de ação.

Descritores: Técnicas de Tipagem Micológica; Candida; Candidemia; Cymbopogon; Óleos; Pediatria.

\section{Abstract}

Introduction: Due to the high mortality rates, candidemia has become a serious health problem in the pediatric reality, especially when considering the increase of infections and the lethality of the cases in groups at risk such as children hospitalized and with deficient immune system. Recently there has been an increase in fungal infections caused by non-albicans species, bringing a new reality to combat these infections, especially since it involves species resistant to conventional therapy. It has been shown that the essential oil of Cymbopogon winterianus has a wide range of pharmacological properties, including antifungal activity. Objective: This work aimed to evaluate the antifungal activity of the essential oil of Cymbopogon winterianus Jowitt ex Bor (Poaceae) against Candida non-albicans isolates of pediatric clinical importance. Materials and Methods: Minimum inhibitory concentration (MIC) and minimum fungicidal concentration (CFM) were determined by broth microdilution techniques. Results: Both the $\mathrm{CIM}_{50}$ and $\mathrm{CFM}_{50}$ of the $C$. winterianus essential oil for the tested isolates were $128 \mu \mathrm{g} / \mathrm{mL}$. Conclusion: This natural product presented antifungal potential in vitro against strains of Candida non-albicans clinically relevant to medical pediatrics. However, more studies are needed to elucidate its mechanism of action.

Descriptors: Mycological Typing Techniques; Candida; Candidemia; Cymbopogon; Oils; Pediatrics.

\section{Resumen}

Introducción: Debido a las altas tasas de mortalidad, la candidemia se ha convertido en un grave problema de salud en la realidad pediátrica, sobre todo cuando se considera el aumento de infecciones y la letalidad de los casos en grupos de riesgo como los niños hospitalizados y con un sistema inmunológico deficiente. Últimamente se han percibido un aumento de infecciones fúngicas provocadas por especies no-albicans, trayendo una nueva realidad para el combate de estas infecciones, especialmente por involucrar a especies resistentes a la terapia convencional. Se ha demostrado que el aceite esencial de Cymbopogon winterianus posee una amplia gama de propiedades farmacológicas, incluyendo actividad antifúngica. Objetivos: Este trabajo buscó evaluar la actividad antifúngica del aceite esencial de Cymbopogon winterianus Jowitt ex Bor (Poaceae) contra aislados de Candida no-albicans de importancia clínica pediátrica. Material y Métodos: La concentración inhibitoria mínima (CIM) y la concentración fungicida mínima (CFM) fueron determinadas por las técnicas de microdilución en caldo. Resultados: Tanto la $\mathrm{CIM}_{50}$ como la $\mathrm{CFM}_{50}$ del aceite esencial de $C$. winterianus para los aislados probados fue de $128 \mu \mathrm{g} / \mathrm{ml}$. Conclusión: Este producto natural presentó potencial antifúngico in vitro contra cepas de Candida no-albicans clínicamente relevante para la pediatría médica. Sin embargo, más estudios son necesarios para elucidar su mecanismo de acción.

Descriptores: Técnicas de Tipificación Micológica; Candida; Candidemia; Cymbopogon; Aceites; Pediatria.

\section{INTRODUÇÃO}

Ao redor do mundo, as infecções fúngicas têm se tornado um grave problema de saúde pública, onde as infecções causadas pelo gênero Candida figuram como a terceira infecção de corrente sanguínea mais frequente. Esta situação adquire maior importância em grupos de risco como os pacientes pediátricos, especialmente aqueles que estão com sistema imunológico comprometido e/ou hospitalizados por longos períodos, onde a candidemia atinge taxas de mortalidades superiores a
$30 \%{ }^{1}$. Estudos sobre a incidência de candidemias na população pediátrica brasileira já mostraram que $C$. albicans é a espécie mais frequentemente isolada, porém, tem ocorrido um aumento no número de candidemias provocadas por espécies não-albicans tais como: $C$. tropicalis, $C$. parapsilosis, $C$. pelliculosa, C. guilliermondii, C. krusei, C. glabrata e C. pararugosa ${ }^{2,3}$.

Estas candidemias provocadas por espécies não-albicans adquirem relevância por apresentarem 
difícil tratamento devido a resistência inata ou adquirida apresentada por estas leveduras contra os agentes antifúngicos tradicionais ${ }^{4}$. Somado a isso, temos a escassez de fármacos disponíveis para um tratamento eficaz de fungemias, onde normalmente apresentam intensa toxicidade ${ }^{5}$, o que denota a importância de sucessivas pesquisas com o intuito de se obter novos compostos que possam ser utilizados contra estas infecções ${ }^{6}$.

Uma valiosa fonte de substâncias com potencial terapêutico está na pesquisa com produtos naturais, especialmente os de origem vegetal. Cymbopogon winterianus Jowitt ex Bor, pertencente a família Poaceae, é uma erva aromática medicinal popularmente conhecida como citronela que já apresentou atividades farmacológicas como antitumoral $^{7}, \quad$ antibacteriana $^{8}$, anticonvulsivante, antioxidante ${ }^{9}$, inseticida ${ }^{10}$ e antimicótica ${ }^{11}$.

Devido às propriedades antifúngicas deste óleo essencial, buscou-se avaliar o potencial antifúngico do óleo essencial de Cymbopogon winterianus contra cepas de Candida não-albicans oriundas de infecções de corrente sanguínea de pacientes pediátricos.

\section{MATERIAL E MÉTODO}

\section{- Substâncias}

O óleo essencial das folhas frescas de $C$. winterianus Jowitt ex Bor foi obtido por hidrodestilação pelo Dr. Paulo Alves Wanderley da Universidade Federal da Paraíba (UFPB). A identificação da planta foi realizada pela Dra. Rita Baltazar de Lima, do Laboratório de Botânica da UFPB. A exsicata foi registrada sob o código JPB 41387 e depositada no Herbário Professor Lauro Pires Xavier na UFPB. A anfotericina B e o fluconazol foram obtidos na Sigma-Aldrich (São Paulo, SP, Brasil), o dimetilsulfóxido (DMSO) e Tween 80 foram comprados na Labsynth Ltda. (Diadema, SP, Brasil). A emulsão dos produtos utilizada nos ensaios antifúngicos foi preparada no momento da execução dos testes. Os produtos foram solubilizados em DMSO, Tween 80 e água destilada, de forma a se obter uma concentração inicial de 1024 $\mu \mathrm{g} / \mathrm{mL}$. A mistura foi agitada durante 3 minutos em um aparelho Vortex (Fanem ${ }^{\circledR}$ Ltda., Guarulhos, SP, Brasil).

\section{- Meios de cultura}

Para testar a atividade biológica dos produtos, foram utilizados o agar Sabouraud dextrose (ASD), adquirido da Difco Laboratories (Detroit, MI, EUA), agar-fubá da HiMédia Laboratories (Mumbai, MH, Índia), e RPMI-1640 com L- glutamina sem bicarbonato de sódio da Sigma-Aldrich (São Paulo, SP, Brasil). Os meios foram preparados e usados de acordo com as instruções dos fabricantes. Os meios foram solubilizados em água destilada e esterilizados em autoclave a $121^{\circ} \mathrm{C}, 1,0 \mathrm{~atm}$ durante $15 \mathrm{~min}$.

\section{- Amostras fúngicas}

Os ensaios foram realizados com seis cepas de Candida não-albicans isoladas de pacientes pediátricos com infecção da corrente sanguinea e duas cepas ATCC como padrão. Duas cepas clínicas de Candida tropicalis (AM-01 e AM-12); duas cepas clínicas de Candida parapsilosis (AM-05 e AM-14) e duas cepas clínicas de Candida pelliculosa (AM-03 e AM-11). As cepas usadas como padrão foram: $C$. tropicalis ATCC-13803 e C. parapsilosis ATCC22019. Estas cepas pertencem à coleção do laboratório de micologia da UFPB e foram mantidas em ASD a $4^{\circ} \mathrm{C}$ até a utilização nos testes.

- Determinação da Concentração Inibitória Mínima (CIM)

A CIM foi determinada pelo método da microdiluição ${ }^{12}$. Culturas de Candida spp. foram semeadas em ASD e incubadas por 24-48 horas em temperatura de $37^{\circ} \mathrm{C}$. Colônias dessas culturas foram suspensas em solução de $0,85 \% \mathrm{NaCl}$ estéril e o inóculo foi padronizado de acordo com a escala 0,5 de McFarland (1-5x10 $\mathrm{UFC} / \mathrm{mL})$. Em placa de 96 poços foram distribuídos meio líquido de RPMI-1640 e óleo essencial de $C$. winterianus em concentrações de 1024 a $0,5 \mu \mathrm{g} / \mathrm{mL}$. A determinação da CIM foi conduzida com aproximadamente $1-5 \times 10^{5} \mathrm{UFC} / \mathrm{mL}$ de microrganismos em cada poço. As placas foram incubadas à $37^{\circ} \mathrm{C}$ por $24-48$ horas. Em $24-48$ horas houve uma observação visual do crescimento fúngico. A CIM foi definida como a menor concentração do óleo que inibiu o crescimento visível da levedura ${ }^{13,14}$. Um controle negativo (sem drogas) foi realizado para confirmar a viabilidade celular fúngica $^{12}$. Um controle de sensibilidade ao Tween 80 e DMSO foi realizado nas mesmas concentrações utilizadas para dissolver os produtos. Houve três independentes experimentos em duplicata em diferentes ocasiões. Os resultados foram expressos como a média aritmética da CIM.

- Determinação da Concentração Fungicida Mínima (CFM)

Para determinar a CFM, $10 \mu \mathrm{L}$ de cada poço sem o crescimento fúngico foi semeado em placas contendo ASD que por sua vez foram incubadas à $37^{\circ} \mathrm{C}$ por $24-48$ horas. A CFM foi considerada como a menor concentração cultivada em placa com SDA em que o crescimento foi inferior a $3 \mathrm{UFC}^{15}$. Um controle negativo (sem drogas) foi realizado para confirmar a viabilidade celular fúngica ${ }^{12}$. Um controle de sensibilidade ao Tween 80 e DMSO foi realizado nas mesmas concentrações utilizadas para dissolver os produtos. Houve três independentes experimentos em duplicata em diferentes ocasiões. Os resultados foram expressos como a média aritmética da CFM.

\section{RESULTADOS}

Para determinação da concentração inibitória 
mínima (CIM) e da concentração fungicida mínima (CFM) do óleo essencial de Cymbopogon winterianus, como visto na Tabela 1, a CIM do óleo essencial testado variou entre 64 e $256 \mu \mathrm{g} / \mathrm{mL}$. A concentração de $256 \mu \mathrm{g} / \mathrm{mL}$ inibiu o crescimento de todas as cepas, enquanto $128 \mu \mathrm{g} / \mathrm{mL}$ foi capaz de inibir acima de $50 \%$ das cepas testadas. A CFM contra os micro-organismos variou entre 128 e 512 $\mu \mathrm{g} / \mathrm{mL}$, sendo esta última concentração fungicida contra todas as cepas testadas. Assim, a $\mathrm{CFM}_{50}$ (concentração fungicida mínima capaz de inibir 50\% das cepas fúngicas) do óleo essencial de $C$. winterianus oil foi $128 \mu \mathrm{g} / \mathrm{mL}$. A razão entre a CFM/CIM demonstrou natureza fungicida do óleo essencial para todas as cepas testadas, exceto contra Candida parapsilosis (AM-05) onde apresentou apenas efeito fungistático. A CIM da anfotericina B variou entre 0.5 e $1 \mu \mathrm{g} / \mathrm{mL}$. Já o fluconazol teve uma CIM entre 0.5 e $8 \mu \mathrm{g} / \mathrm{mL}$. Os resultados para o ensaio controle não apresentou inibição do crescimento fúngico.

Tabela 1: CIM, CFM, CFM/CIM e efeito do óleo essencial de Cymbopogon winterianus e CIM da anfotericina B e fluconazol contra cepas das espécies de Candida não-albicans.

\begin{tabular}{|c|c|c|c|c|c|c|c|}
\hline \multirow[t]{2}{*}{ Leveduras } & \multicolumn{4}{|c|}{$\begin{array}{l}\text { Óleo essencial de C. winterianus } \\
(\mu \mathrm{g} / \mathrm{mL})\end{array}$} & \multirow{2}{*}{$\begin{array}{c}\begin{array}{c}\text { Anf } \\
(\mu \mathrm{g} / \mathrm{mL})\end{array} \\
\mathrm{CIM}\end{array}$} & \multirow{2}{*}{$\begin{array}{c}\begin{array}{c}\text { Flc } \\
(\mu \mathrm{g} / \mathrm{mL})\end{array} \\
\mathrm{CIM}\end{array}$} & \multirow[t]{2}{*}{ Controle $^{\mathrm{a}}$} \\
\hline & CIM & CFM & $\frac{\text { CFM }}{\text { CIM }}$ & Efeito & & & \\
\hline \multicolumn{8}{|l|}{ C.tropicalis } \\
\hline $\begin{array}{l}\text { ATCC- } \\
13803\end{array}$ & 128 & 128 & 1:1 & Fungicida & 0.5 & 0.5 & + \\
\hline AM-01 & 128 & 128 & $1: 1$ & Fungicida & 1 & 1 & + \\
\hline AM-12 & 128 & 256 & $2: 1$ & Fungicida & 0.5 & 0.5 & + \\
\hline \multicolumn{8}{|c|}{ C.parapsilosis } \\
\hline $\begin{array}{l}\text { ATCC } \\
22019\end{array}$ & 128 & 128 & $1: 1$ & Fungicida & 0.5 & 0.5 & + \\
\hline AM-o5 & 128 & 512 & $4: 1$ & Fungistático & 1 & 1 & + \\
\hline AM-14 & 256 & 512 & $2: 1$ & Fungicida & 0.5 & 4 & + \\
\hline \multicolumn{8}{|c|}{ C.pelliculosa } \\
\hline AM-03 & 256 & 256 & $1: 1$ & Fungicida & 1 & 8 & + \\
\hline AM-11 & 64 & 128 & $2: 1$ & Fungicida & 1 & 8 & + \\
\hline
\end{tabular}

\section{DISCUSSÃO}

Infecções de correntes sanguíneas provocadas por Candida representam um importante causa de morte em pacientes pediátricos hospitalizados em todo o mundo ${ }^{16}$. C. albicans permanece como a mais frequente espécie causadora deste tipo de infecção. Porém, outras espécies com reduzida susceptibilidade aos agentes antifúngicos como C. tropicalis, C. parapsilosis, C. glabrata, $C$. krusei e C. guilliermondii estão sendo constantemente encontradas como o agente da fungemia ${ }^{17}$.

Tanto as plantas medicinais quanto seus fitoconstituintes são uma valiosa fonte de novos fármacos para o tratamento de diversas doenças ${ }^{18}$. Os óleos essenciais vegetais, ricos em monoterpenos e sesquiterpenos são umas destas fontes de potenciais agentes terapêuticos, o que atribui valor comercial a estes produtos ${ }^{19}$.

Em outros estudos, o óleo essencial de $C$. winterianus apresentou valores elevados de CIM $(600-1250 \mu \mathrm{g} / \mathrm{mL})$ contra cepas de Candida albicans isoladas de pacientes adultos ${ }^{11,20}$. Esta diferença pode ocorrer por causa da origem e linhagem dos microorganismos testados, uma vez que estas leveduras podem apresentar uma resistência inata ou adquirida de acordo com a situação do paciente.

A atividade antimicrobiana de uma determinada substância pode ser interpretada de acordo com Sartoratto et al. ${ }^{21}$ (2004): forte/boa atividade (CIM: $<500 \mu \mathrm{g} / \mathrm{mL}$ ) e Morales et al. ${ }^{22}$ (2008): forte/boa atividade (CIM: $<100 \mu \mathrm{g} / \mathrm{mL}$ ); atividade moderada (CIM: $100-500 \mu \mathrm{g} / \mathrm{mL}$ ); fraca atividade (CIM: $500-1000 \mu \mathrm{g} / \mathrm{mL}$ ); e produto inativo/sem atividade antimicrobiana (CIM: >1000 $\mu \mathrm{g} / \mathrm{mL})$. De acordo com Satoratto et al. ${ }^{21}$ o óleo essencial de $C$. winterianus demonstrou uma forte atividade antifúngica contra Candida não-albicans pois seus valores de CIM são infereiores a 500 $\mu \mathrm{g} / \mathrm{mL}$. Na literatura, o óleo essencial de $C$. winterianus já demonstrou ser ativo contra bactérias e fungos $^{23}$, incluindo Candida albicans e nãoalbicans $^{11,20}$.

Anfotericina B e fluconazol são amplamente utilizados como os agentes terapêuticos nos casos de candidemia em pacientes pediátricos devido a sua eficácia, ainda que a toxicidade da anfotericina B limite seu uso ${ }^{24}$. De acordo com os resultados obtidos e com os critérios de Morales et al. ${ }^{22}$, anfotericina B e fluconazol apresentaram ótimo efeito antifúngico contra Candida não-albicans, uma vez que seus valores de CIM foram inferiores a $100 \mu \mathrm{g} / \mathrm{mL}$.

De acordo com Hafidh et al. ${ }^{25}$ a relação CFM/CIM é usada para especificar a natureza do efeito antimicrobiano contra um patógeno particular. Quando a relação CFM/CIM está entre 1/1 e 2/1, o produto químico é considerado fungicida. Por outro lado, se a razão é> 2:1, é mais provável que seja fungistático.

Neste estudo, a razão CFM/CIM do óleo essencial de $C$. winterianus apresentou efeito fungicida contra a maioria das cepas testadas, exceto contra Candida parapsilosis (AM-05), onde agiu como fungistático. O uso extensivo de agentes fungistáticos tem promovido o aparecimento de cepas resistentes, compromotendo o sucesso da terapia antifúngica. Por este motivo, substâncias fungicidas são desejáveis pois tem menores chances de provocar o aparecimento de resistência nas cepas clínicas ${ }^{26}$.

Os achados deste trabalho são relevantes e encorajadores para conduzir mais estudos envolvendo o óleo essencial de $C$. winterianus, especialmente sobre a elucidação do seu mecanismo de ação.

\section{CONCLUSÃO}

O presente estudo demonstrou que o óleo essencial de Cymbopogon winterianus possui atividade antifúngica significativa contra os isolados de Candida não-albicans de importância clínica pediátrica. Desta forma, este trabalho possui 
relevância por contribuir com o desenvolvimento de novas alternativas para o arsenal terapêutico existente. Contudo, são necessários mais estudos para investigar o mecanismo de ação exercido pelo óleo essencial de Cymbopogon winterianus sobre a célula fúngica.

\section{AGRADECIMENTOS}

O presente trabalho foi realizado com apoio da Coordenação de Aperfeiçoamento de Pessoal de Nível Superior - Brasil (CAPES), Conselho Nacional de Desenvolvimento Científico e Tecnológico (CNPq) e Universidade Federal da Paraíba (UFPB).

\section{REFERÊNCIAS}

1. Steinbach WJ. Pediatric invasive candidiasis: epidemiology and diagnosis in children. J Fungi (Basel). 2016;2(1):5.

2. Ruiz LS, Khouri S, Hahn RC, da Silva EG, de Oliveira VK, Gandra RF et al. Candidemia by species of the Candida parapsilosis complex in children's hospital: prevalence, biofilm production and antifungal susceptibility. Mycopathologia. 2013;175(3-4):231-39.

3. Oliveira VKP, Ruiz LS, Oliveira NAJ, Moreira D, Hahn RC, Melo ASA et al. Fungemia caused by candida species in a Children's Public Hospital in the city of São Paulo, Brazil: study in the period 2007-2010. Rev Inst Med Trop Sao Paulo. 2014;56(4):301-5.

4. Morace G, Borghi E, Iatta R, Amato G, Andreoni $S$, Brigante $G$ et al. Antifungal susceptibility of invasive yeast isolates in Italy: the GISIA3 study in critically ill patients. BMC Infect Dis. 2011;11:130.

5. Khan SMA, Malik A, Ahmad I. Anti-candidal activity of essential oils alone and in combination with amphotericin B or fluconazole against multidrug resistant isolates of Candida albicans. Med Mycol. 2012;50(1):33-42.

6. Svetaz L, Aguero MB, Alvarez S, Luna L, Feresin $\mathrm{G}$, Derita $\mathrm{M}$ et al. Antifungal activity of Zuccagnia punctata Cav.: evidence for the mechanism of action. Planta Med. 2007;73(10):1074-80.

7. Ganjewala D, Silviya S, Khan HK. Biochemical composition and antibacterial activities of Lantana Camera plants with yellow, lavender, red and white flowers. EurAsia J BioSci. 2009;3:69-77.

8. Scazzocchio F, Garzoli S, Conti C, Leone C, Renaioli C, Pepi F et al. Properties and limits of some essential oils: chemical characterisation, antimicrobial activity, interaction with antibiotics and cytotoxicity. Nat Prod Res. 2016;30(17):1909-18.

9. Silva MR, Ximenes RM, da Costa JG, Leal LK, de Lopes AA, Viana GS. Comparative anticonvulsant activities of the essential oils (EOs) from Cymbopogon winterianus Jowitt and
Cymbopogon citratus (DC) Stapf. in mice. Naunyn Schmiedebergs Naunyn Schmiedebergs Arch Pharmacol. 2010;381(5):415-26.

10.Silva CT, Wanderley-Teixeira V, Cunha FM, Oliveira JV, Dutra KA, Navarro DM et al. Biochemical parameters of Spodoptera frugiperda (J. E. Smith) treated with citronella oil (Cymbopogon winterianus Jowitt ex Bor) and its influence on reproduction. Acta Histochem. 2016;118(4):347-52.

11.Oliveira WA, Pereira FO, Luna GCDG, Lima IO, Wanderley PA, Lima RB et al. Antifungal activity of Cymbopogon winterianus Jowitt ex Bor against Candida albicans. Braz J Microbiol. 2011;42(2):433-41.

12.Eloff JN. A sensitive and quick microplate method to determine the minimal inhibitory concentration of plant extracts for bacteria. Planta Med. 1998;64(8):711-13.

13.Hadacek F, Greger H. Testing of antifungal natural products: methodologies, comparability of results and assay choice. Phytochem Anal. 2000;11(3):137-47.

14.CLSI. Clinical and laboratory standards institute. protocol M27-A3. Reference method for broth dilution antifungal susceptibility testing of yeasts. 3ed. Wayne, PA, USA. 2008.

15.Espinel-Ingroff A, Chaturvedi V, Fothergill A, Rinaldi MG. Optimal testing conditions for determining MICs and minimum fungicidal concentrations of new and established antifungal agents for uncommon molds: NCCLS collaborative study. J Clin Microbiol. 2002;40(10):3776-81.

16.Falagas ME, Roussos N, Vardakas KZ. Relative frequency of 3 albicans and the various non-albicans Candida spp among candidemia isolates from inpatients in various parts of the world: a systematic review. Int $\mathrm{J}$ Infect Dis. 2010;14(11):e954-66.

17.Nucci M, Queiroz-Telles F, Alvarado-Matute T, Tiraboschi IN, Corte J, Zurita $J$ et al. Epidemiology of candidemia in Latin America: a laboratory-based survey. Plos One. 2013;8(3):e59373.

18.Simões ER, Santos EA, de Abreu MC, Silva JN, Nunes NM, da Costa MP et al. Biomedical properties and potentiality of Lippia microphylla Cham. and its essential oils. J Intercult Ethnopharmacol. 2015;4(3):256-63.

19. Bilia AR, Santomauro F, Sacco C, Bergonzi MC, Donato R. Essential Oil of Artemisia annua L.: An Extraordinary Component with Numerous Antimicrobial Properties. Evid Based Complement Alternat Med. 2014; 2014:159819.

20.Duarte MCT, Figueira G M, Sartoratto A, Rehder VLG, Delarmelina C. Anti-Candida activity of Brazilian medicinal plants. J Ethnopharmacol. 2005;97(2):305-11. 
21.Sartoratto A, Machado ALM, Delarmelina C, Figueira GM, Duarte MCT, Rehder VLG. Composition and antimicrobial activity of essential oils from aromatic plants used in Brazil. Braz J Microbiol. 2004;35(4):275-80.

22. Morales G, Paredes A, Sierra P, Loyola LA. Antimicrobial activity of three baccharis species used in the traditional medicine of Northern Chile. Molecules. 2008;13(4):790-94.

23. Oliveira WA, Arrua JMM, Wanderley PA, Lima RB, Lima EO. Effects of the essential oil of Cymbopogon winterianus against Candida albicans. Rev Pan-Amaz Saude. 2015;6(3):21-6.

24.Tragiannidis A, Tsoulas C, Groll AH. Invasive candidiasis and candidaemia in neonates and children: update on current guidelines. Mycoses 2015;58(1):10-21.

25.Hafidh RR, Abdulamir AS, Vern LS, Bakar FA, Abas F, Jahanshiri F et al. Inhibition of growth of highly resistant bacterial and fungal pathogens by a natural product. Open Microbiol J. 2011;5:96-106.

26. Monk BC, Goffeau A. Outwitting multidrug resistance to antifungals. Sci. 2008;321(5887):367-69.

\section{CONFLITO DE INTERESSES}

Os autores declaram não haver conflitos de interesse.

\section{AUTOR PARA CORRESPONDENCIA}

\section{Hermes Diniz Neto}

hermes.dn@hotmail.com
Submetido em 17/01/2019

Aceito em 12/03/2019 